\title{
IMPORTANT ASPECTS OF HUMAN BLOOD EXPOSURE IN THE RADIO AND MICROWAVE FIELD
}

\author{
A. M. SILAGHI ${ }^{1}$, U. L. ROHDE ${ }^{2}$, A. K. PODDAR ${ }^{2}$, H. SILAGHI ${ }^{1}$, T. I. ILIAS ${ }^{3}$, O.C. FRATILA ${ }^{3}$ \\ ${ }^{1}$ University of Oradea, Faculty of Electrical Engineering and Information Technology, University Street, 1, 410087 \\ Oradea, Romania, ${ }^{2}$ Synergy Microwave Corp. New Jersey, NJ, USA and University of Oradea, University Street, 1, \\ 410087 Oradea, Romania, ${ }^{3}$ University of Oradea, Faculty of Medicine and Pharmacy, Romania \\ E-mail: hsilaghi@uoradea.ro, masilaghi@uoradea.ro, akpoddar@synergymwave.com, akpoddar@uoradea.ro, \\ tiberia_ilias@yahoo.com,ovidiufr@yahoo.co.uk
}

\begin{abstract}
To expose humans to microwave emissions damages biological welfare. For measuring the dielectric properties of biological tissues, it was studied whether current safety standard recommendations are inappropriate as far as human blood function is concerned. Thirty age and gender matched blood samples will serve as control. For increased accuracy detection of any possible alteration and to help orientate towards the details that are to be studied ultra-structurally, a special stain was used. An electronic microscope analysis will be performed on all the blood samples after specific preparation to see whether such microwave exposure might affect the ultra-structure of the blood cells. After modeling and sectioning the blocks were contrasted using acetate-uranyl and lead-citrate and study them with an electronic microscope. The obtained images are processed using Viewer Imaging Analysis software for further analysis. In the end, scanning microscopy must be used in order to detect any surface dimorphisms that might appear due to the microwave exposure.
\end{abstract}

Keywords: Exposure to microwave field emissions, microscope analysis, modeling, simulation, ultrastructure of the human blood and biological effects

\section{INTRODUCTION}

Electronic gadget devices have become essential components of daily life. However, their deleterious effects on the body, particularly on the nervous system, are well known.

Electromagnetic fields have various chemical effects, including causing deterioration in large molecules in cells and imbalance in ionic equilibrium.

The effects of microwaves might be divided in next categories: alterating of nutritive value, health effects of exposure and effects that can lead to cancer $[2,8,10]$.

Different scientists have studied the effects of microwave exposure on living organisms, from biological point of view $[1,4,10]$. It is understood that the effects of electromagnetic field are varied and reliant on the strength, frequency, and duration of the electromagnetic field exposures [11-12]. Lifetime exposure to electromagnetic field is fetching the topic of notable scientific inquiry since it has the impending to source vital alterations and injurious effects in biological systems. The biological impacts of electromagnetic field can be classified as thermal and non-thermal. Thermal effects are associated with the heat created by EM-field in a definite region. This mechanism happens through transference in temperature originating from radio frequency fields. It is possible that every interaction between radio frequency fields and living tissues causes an energy transfer consequential in a rise in temperature. Exposures to electromagnetic fields can impair biological tissues by inducing variations, which can be elucidated in terms of thermal or non-thermal mechanisms. Thermal effects can occur with the conversion and absorption of heat by the body's electromagnetic energy. The Increased body temperature is stabilized and lessened by blood circulation. Although non-thermal effects do not raise the body temperature sufficiently to impair the structure of tissues, their effects can still be seen as an increase in free radical production in tissues [12].

Few studies were made on animals to see if blood was affected by exposure to microwave that exceed current safety standard recommendations $[4,5]$. Therefore, it is useful to verify whether current safety standard recommendations are inappropriate as far as human blood function is concerned.

The total current through blood can be described by [6]

$$
\begin{gathered}
I(t)=\sum_{i=1}^{n}\left\{\left(G_{c i} V+G_{d i} V\right)+\left(C_{c i} V \frac{d V}{d t}+C_{d i} V \frac{d V}{d t}\right)\right\}(1) \\
I(t)=\sum_{i=1}^{n}\left\{\left(\sigma_{c i}+\sigma_{d i}\right)+i \omega\left(\varepsilon_{c i}+\varepsilon_{d i}\right)\right\} \frac{V}{d} A
\end{gathered}
$$

where $I_{c i}$ and $I_{d i}$ are the conduction and displacement currents that carried by any particle $(i)$ in the blood, $\sigma_{c i}$ and $\sigma_{d i}$ represent electrical conductivity and displacement conductivity, $\varepsilon_{c i}$ and $\varepsilon_{d i}$ denote the conduction dielectric constant and displacement dielectric constant of any particle $(i), G$ is the conductance, $\varepsilon_{0}=8.855 \times 10^{-12} \mathrm{~F} / \mathrm{m}$ is the reference dielectric constant, [13].

We describe blood by using complex admittance, $Y^{*}$ and complex conductivity $\sigma^{*}$

$$
Y^{*}=\sum_{i=1}^{n}\left\{\left(G_{c i}+G_{d i}\right)+j \omega\left(C_{c i}+C_{d i}\right)\right\}
$$




$$
\begin{gathered}
Y^{*}=\sum_{i=1}^{n}\left\{\left(\sigma_{c i}+\sigma_{d i}\right)+j \omega\left(\varepsilon_{c i}+\varepsilon_{d i}\right)\right\} \frac{A}{d} \\
\sigma^{*}=\sum_{i=1}^{n}\left\{\left(\sigma_{c i}+\sigma_{d i}\right)+j \omega\left(\varepsilon_{c i}+\varepsilon_{d i}\right)\right\}
\end{gathered}
$$

From (1) and (2)

$$
I(t)=\sum_{i=1}^{n}\left\{\left(\varepsilon_{c i}+\varepsilon_{d i}\right)-i \frac{\left(\sigma_{c i}+\sigma_{d i}\right)}{\omega \varepsilon_{\varepsilon}}\right\} j \omega \varepsilon_{0}\left(\frac{A}{d}\right)
$$

The complex relative permittivity is given by

$$
\begin{gathered}
\varepsilon^{\star}=\sum_{i=1}^{n}\left(\varepsilon_{c i}+\varepsilon_{d i}\right)-j \frac{\left(\sigma_{c i}+\sigma_{d i}\right)}{\omega \varepsilon_{s}}=\varepsilon_{r}^{r}-i \varepsilon_{r}^{" r} \\
\varepsilon^{\star}=\left[\varepsilon_{r}-j \frac{\sigma}{\omega \varepsilon_{s}}\right]=\varepsilon_{r}^{r}-j \varepsilon_{r}^{\prime \prime}
\end{gathered}
$$

Where $\varepsilon_{r}=\sum_{i=1}^{n}\left(\varepsilon_{c i}+\varepsilon_{d i}\right)$ and $\varepsilon_{r}^{\mathrm{w}}=\sum_{i=1}^{n} \frac{\left(\sigma_{c i}+\sigma_{d i}\right)}{\omega \varepsilon_{0}}$

From (1) to (8), bio-impedance relations can offer an important method for investigation of internal structure of blood [6].

From (5) and (6), it is obvious that as a dielectric dispersion is traversed by changing the frequency of measurement, the change in conductivity is directly proportional to the change in permittivity. So, the permittivity is increasing and also the temperature of blood is increasing and the blood cells will have altered structure.

\section{METHODS OF EXPOSER INVESTIGATION}

In order to investigate whether the electromagnetic fields associated with electronic gadgets interfere with blood cell, this study involved blood samples from 40 otherwise healthy subjects who were asked to attend the Clinical County Hospital from Oradea, Romania. All participants had had their history taking a physical examination to detect symptoms and signs of any disease. All these data was recorded and further analysis was assessed.

After informed consent, a blood sample was taken from all patients using syringes and it was later stored in sealed test tubes at $4^{\circ} \mathrm{C}$ for preventing coagulation $[3,5]$. Afterwards the blood from the patients was exposed to a microwave field using the laboratory desk, as depicted in Figure 1.

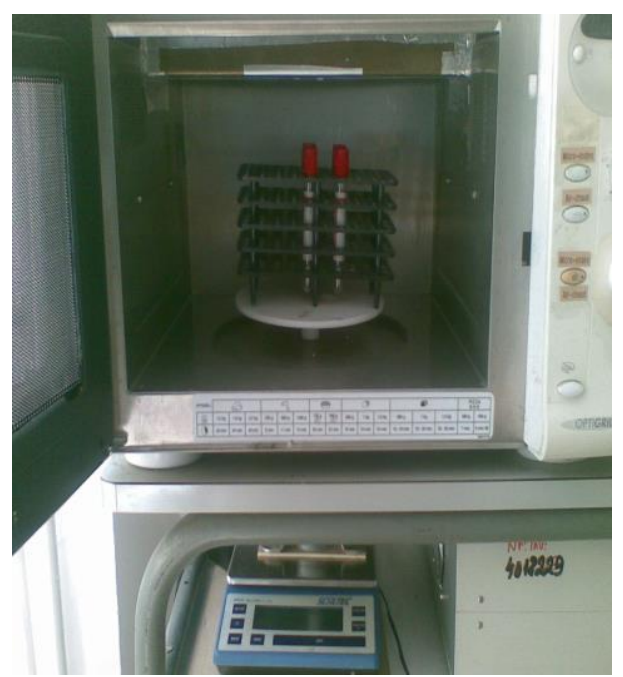

Figure 1. Microwave laboratory desk

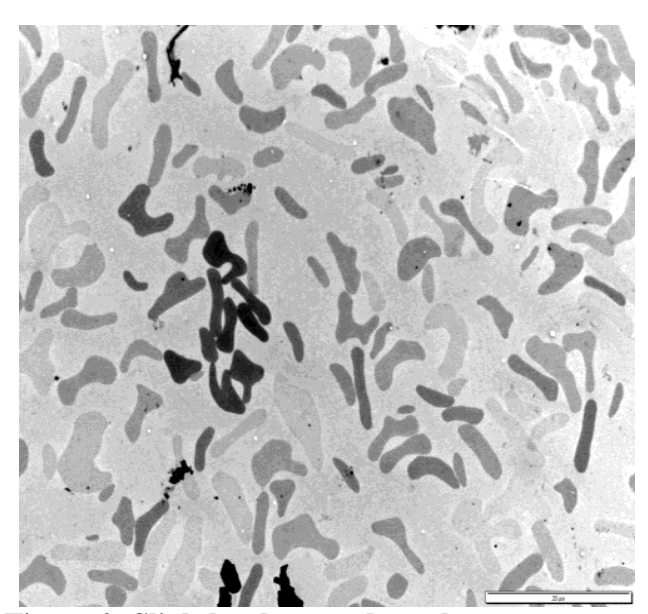

Figure 2. Slightly abnormal erythrocytes aspect

The experimental model for microwave processes is composed from one microwave generator at 2450 $\mathrm{MHz}$, and $1 \mathrm{KW}$ variable power as presented, which is connected with interface to $\mathrm{PC}$ and printer; the magnetron type is TOSHIBA 2M248, from Microwave Techniques laboratory, University of Oradea, Figures 2, 3 and 4 show the slightly abnormal erythrocytes, basophiles cells with normal ultrastructural and neutrophil cells with normal ultra-structural aspects.

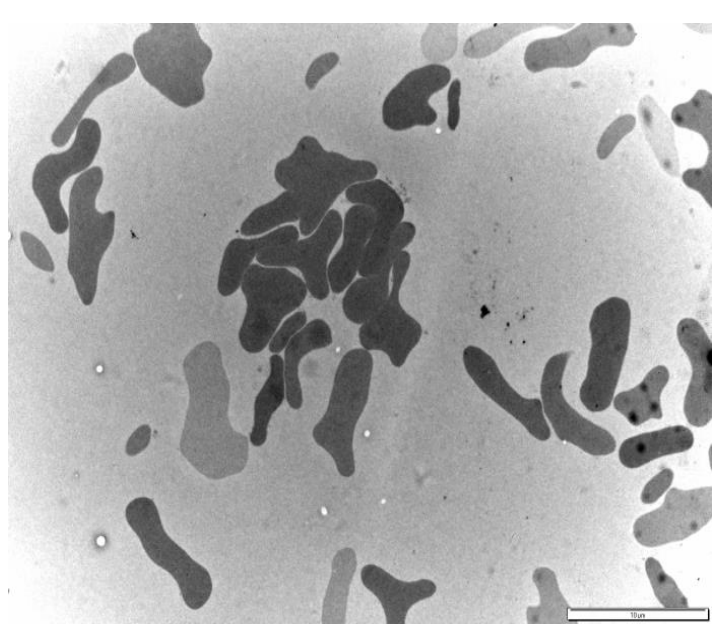

Figure 3. Basophiles cells with normal ultrastructural aspect 
The electronic balance for the measure of weight is for maximum $3100 \mathrm{~g}$, with $0.1 \mathrm{~g}$ precision and is connected to PC. It is predicted also a dummy load connected to water network from laboratory and electrical generator for the microwave/hot-air (750W).

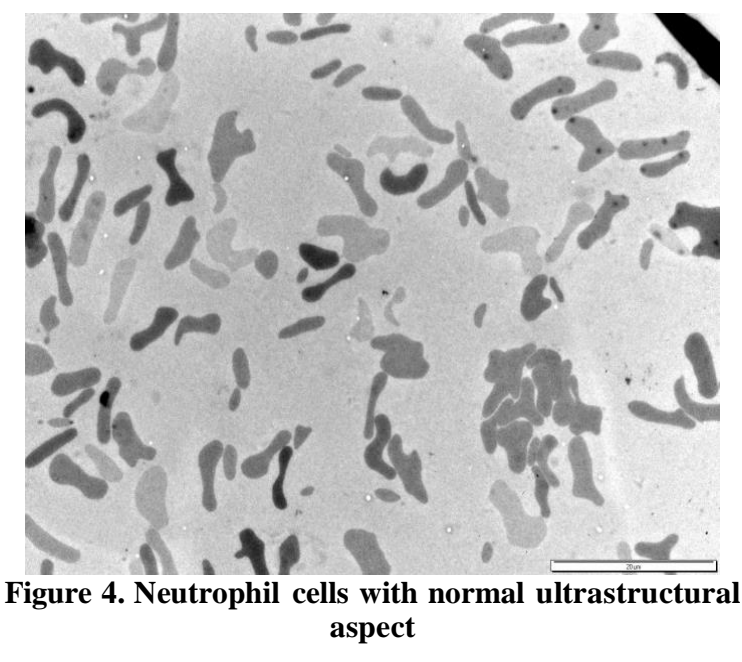

The following assumptions have been considered: working frequency $2450 \mathrm{MHz}$, possibility to measure reflected and transmitted power and homogenous electromagnetic field distribution to assure an accurate exposure.

The temperature responses during each experiment are recorded. Thirty age and gender matched blood samples will serve as control [6,7]. So, in the end two groups were formed:

- Group 1, control blood, not treated with microwaves, called CHB (control human blood);

- Group 2, microwave treated blood called MTHB (microwave treated human blood).

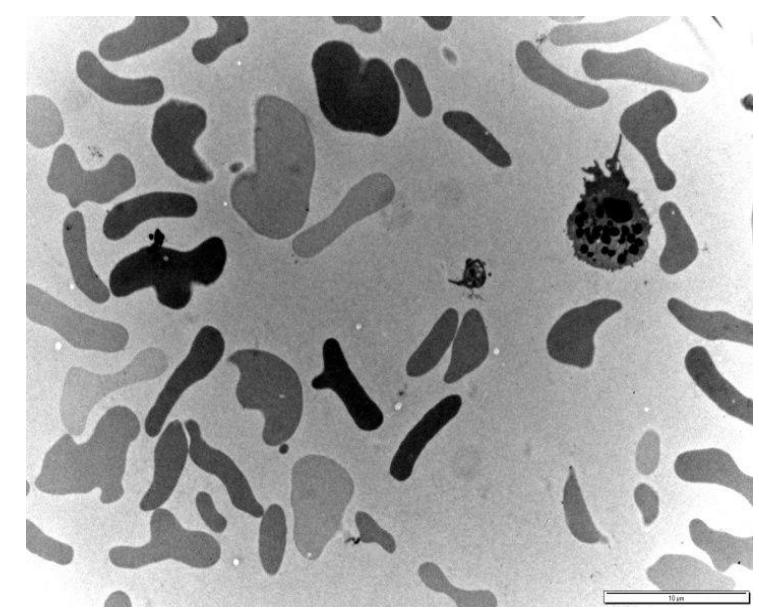

Figure 5. Neutrophil cells with normal ultrastructural aspect

An electron microscope analysis was performed on all the blood samples after specific preparation to see whether such microwave exposure might affect the ultrastructure of the blood cells.

After separating the serum from the blood cells, the latter was prefixed with $2.7 \%$ glutaraldehyde solution in $0.1 \mathrm{M}, \mathrm{pH} 7.2$ phosphate buffer resulting tiny tissue blocks $[9,14]$. The post fixing process was done with $1 \%$ osmium tetroxide $(\mathrm{OsO} 4)$.

The water extraction was done gradually, in short times to ensure that the process is not followed by structural alterations. The specimen encapsulation was made using special plastic capsules followed by a polymerizing process (temperature 50-60 C degrees for 72 hours) from which some hard transparent blocks with black pieces inside have been obtained.

It is to be mentioned that at every liquid changing during the processing, the blood samples were spinned at 1000 rotations/minute in order to remove the supernatant. From the afore mentioned blocks some semifine sections have been obtained measuring $500 \mathrm{~nm}$ for light microscopy. Later from the selected zones of the same blocks some ultrathin sections have been cut, measuring 40-60 nm for ultrastructural assessment. The sections were made using a Leica UC6 ultramicrotom which has DDK diamond knives.

Then the second fixing agent $1 \% \mathrm{OsO} 4$ has been added in $0.1 \mathrm{M}$ phosphate buffer at a $7.4 \mathrm{pH}$ for 15 minutes. Using the aforementioned fixing procedure we intended to stop the metabolic processes of the tissue, to preserve the fine structure of the cells for future preparing and optimal visualizing with the transmission electron microscope [15-18]. For the inclusion process an epoxidic pitch-Epon812 has been used. For dehydration acetone in water solution in rising concentrations has been used.

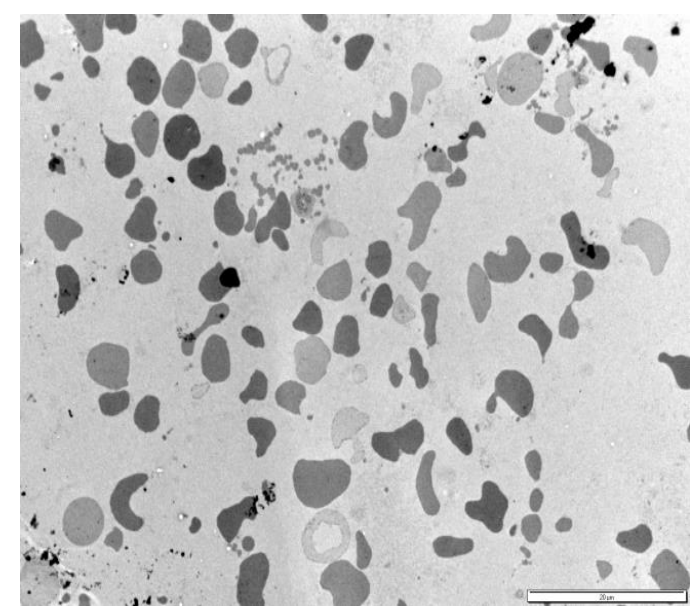

Figure 6. PMN cells with degranulation process

After modeling and sectioning the blocks they were contrasted and studied with an electron microscope. The pictures were captured using a Megaview III camera. The obtained images were processed using Viewer Imaging Analysis software for further analysis.

\section{EXPERIMENTS RESULTS}

For both groups plasma membranes, cytoplasm matrix and other components of peripheral blood have been studied. 
The overview images obtained from the semi fine sections showed the general situation of red blood cells in CHB group, compared to the red blood cells images in the MTHB group. We observed that in MTHB group the red blood cells tend to became spherical, phenomena probably due to the alteration of the permeability membrane capacity.

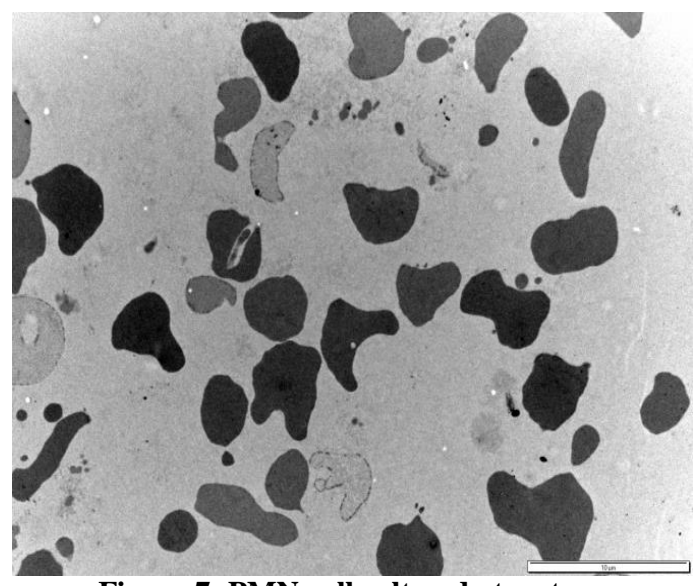

Figure 7. PMN cells altered structure

The ultrastructural images obtained at successive magnification showed the normal conformation of the red blood cell population in $\mathrm{CHB}$ group, with electrondense cytoplasm due to the presence of hemoglobin.

In CHB group the red blood cells are almost normal in shape, the majority having the shape of a biconcave disk from side view. There are also slightly abnormal erythrocytes probably due to a long period from blood collection till fixing processes (Figure 2).

Some basophiles (Figure 3) and neutrophil (Figure 4 and Figure 5) cells with normal ultrastructural aspect can be observed in the structure of leucocytes.

In the MTHB group all the erythrocytes are oval arround, many of them having a diluted content and some of them being destroyed (hemolysis) so that fragments from them are dispersed in the plasma serum.

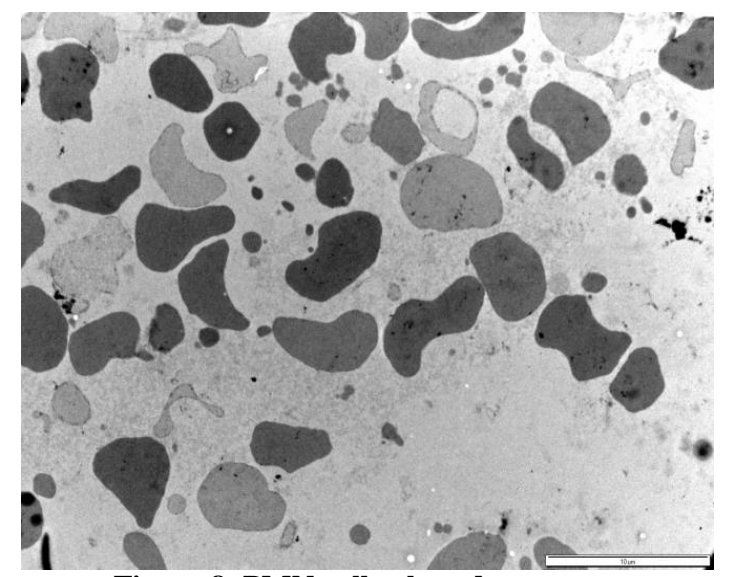

Figure 8. PMN cells altered structure
It can be observed that the PMN cells have altered structure (Figure 6), abnormal plasmatic membrane so that they suffer a degranulation process (Figure 7 and Figure 8).

\section{CONCLUSION}

The results of the present study indicate that the applied microwaves have induced alterations of the ultrastructure of the human blood cells and organelles, processes that appear especially due to the alteration of membrane permeability capacity.

The ultrastructural assessment done in this research comprised especially the modifications which appear in the human blood and red blood cells distinctively before and after microwave treatment.

The pictures were captured using a Megaview III camera and the obtained images were processed using Viewer Imaging Analysis software for further analysis.

The original contributions of this paper are: after microwave treatment in the MTHB group all the erythrocytes are oval around, many of them having a diluted content and some of them being destroyed (hemolysis) so that fragments from them are dispersed in the plasma serum. The PMN cells have altered structure, abnormal plasmatic membrane so that they suffer a degranulation process after microwave exposure.

Future studies are needed for a better clarification of the action mechanisms and evolution of our blood when it is submitted to microwave exposure.

\section{ACKNOWLEDGMENTS}

The authors would like to direct their warmest thanks to Oradea University and Synergy Microwave for sponsoring the research activities.

\section{REFERENCES}

[1] A. De Sabata, L. Maketovits, I. Peter, U.L.Rohde, M.A. Silaghi, "Metamaterial based high impedance surface with band-pass frequency response", in Materials Science Forum,vol.721,2012, Tarns Tech Publications,Switzerland,ISBN 978-3-901509-919,vol.23,Nr.1,pp.60-64.

[2] A. Silaghi, A.De Sabata, M. A. Silaghi , Testing immunity to portable transmitters with helical antennas: Key concepts, 2016 Ieee 22nd International Symposium For Design and Technology and Electronic Packaging (SIITME) 2016.

[3] De Sabata, A., Matekovits, L., Silaghi, A.M., Rohde, U.L., Silaghi, M.A., Investigation on surface waves related properties of some periodic structures, Proceedings of the 12th International Conference on 
Microwave and High Frequency Heating, AMPERE 2009, pp. 364-367.

[4] O. Fratila, A. Maghiar, Tiberia Ioana Ilias, M.A. Silaghi, "Analysis of the Colonoscopic Findings in patients with the acute Gastrointestinal Tract Bleeding", in Journal of Computer Scinence and Control Systems, University of Oradea, Romania, ISSN 1844-6043, pp. 135-140,2008.

[5] Silaghi, A.M. and Rohde, L.U., "Numerical Modelling of Phenomenon in Microwave Applicator", The Scientific Bulletin of Electrical Engineering Faculty, Targoviste, Romania, ISSN 1843-6188, 2008, pp.62-66.

[6] S. Abdalla, S. S. Al-ameer, and S. H. Al-Magaishi, "Electrical properties with relaxation through human blood", BIOMICROFLUIDICS pp. 0341011 to 034101-15, American Institute of Physics, 2010.

[7] P. Izzo1, A. Spagnuolo1, A. Manicone1, P. Nazzaro, and V.M. Lauta, Journal Clinical Hemorheology and Microcirculation 21, 3 _1999.

[8] Fragopoulou AF, Koussoulakos SL, Margaritis LH. Cranial and postcranialskeletal variations induced in mouse embryos by mobile phone radiation. Pathophysiology 2010.

[9] Rai, S. et al. Effect of modulated microwave frequencies on the physiology of a cyanobacterium. Anabaena doliolum Electromagn. Biol. Med. 18, 221-232 (1999).

[10] Banik, S., Bandyopadhyay, S. \& Ganguly, S. Bioeffects of microwave - A brief review. Bioresour. Technol. 87, 155-159 (2003).

[11] L.J. Challis, "Mechanisms for interaction between RF fields and biological tissue", Bioelectromagnetics (2005), pp. S 98-106.

[12] Veronesi, P., Leonelli, Cristina, Moscato, U., Cappi, A., and Figurelli, O, "Non-incineration microwave assisted sterilization of medical waste", Journal of Microwave Power \& Electromagnetic Energy, Vol. 40, No. 4, 2007, pp.211-218.

[13] A. Silaghi and A. De Sabata, „Emissions and Immunity EMC Testing at Continental Automotive Timisoara", Journal of Electrical and Electronic Engineering (JEEE), vol.11, nr.1, May 2018, pp. 3339.

[14] Hayat, M.A., Principles and techniques electron microscopy, Biological Appl. Fourth Edition, Ed. Cambridge Univ. Press, 2000.

[15] Metaxas, A. C., Meredith, R. J., Industrial Microwave Heating, Peter Peregrinus Ltd., London, U.K., 1983.

[16] O. Fratila, M.Tantau, "Cleaning and Disinfection in Gastrointestinal Endoscopy: Current Status in Romania", *Published in abstract at the 26th National Symposium of Gastroenterology, Hepatology and Digestive Endoscopy, Timisoara 2005. 